\title{
Change Detection over Sokolov Open-Pit Mining Area, Czech Republic, Using Multi-Temporal HyMAP Data (2009-2010)
}

\author{
S. Adar*a, G. Notesco ${ }^{\text {b }}$, A. Brook ${ }^{\text {b }}$ I. Livne ${ }^{\mathrm{b}}$, P. Rojik ${ }^{\mathrm{c}}$, V. Kopackova $^{\mathrm{d}}$, K. Zelenkova ${ }^{\mathrm{d}}$, J. Mišurec ${ }^{\mathrm{d}}$, \\ A. Bourguignon ${ }^{\mathrm{e}}$, S. Chevrel ${ }^{\mathrm{e}}$, C. Ehrler ${ }^{\mathrm{f}}$, C. Fisher ${ }^{\mathrm{f}}$, J. Hanuš ${ }^{\mathrm{g}}$, Y. Shkolnisky ${ }^{\mathrm{h}}$ and E. Ben Dor ${ }^{\mathrm{b}}$ \\ ${ }^{a}$ Porter School of Environmental Studies at Tel Aviv University, Tel Aviv, Israel \\ ${ }^{\mathrm{b}}$ Department of Geography and Human Environment, Tel Aviv University, Israel \\ 'Sokolovská uhelná a. s., Staré nám. 69, Sokolov, Czech Republic \\ ${ }^{\mathrm{d} C z e c h}$ Geological Survey, Klárov 3, 118 21, Prague 1, Czech Republic \\ ${ }^{\mathrm{e}}$ BRGM, Mineral Resource division Orleans, France \\ ${ }^{\mathrm{f}}$ German Aerospace Center, German Remote Sensing Data Center, Dept. Land Applications, \\ Wessling, Germany \\ ${ }^{g}$ Institute of Systems Biology and Ecology of the Academy of Sciences of the Czech Republic, Na \\ Sádkách 7, České Budějovice, Czech Republic \\ ${ }^{\mathrm{h}}$ Department of Applied Mathematics, Tel-Aviv University, Israel \\ "simon.adar@gmail.com
}

\begin{abstract}
Two HyMap images acquired over the same lignite open-pit mining site in Sokolov, Czech Republic, during the summers of 2009 and 2010 (12 months apart), were investigated in this study. The site selected for this research is one of three test sites (the others being in South Africa and Kyrgyzstan) within the framework of the EO-MINERS FP7 Project (http://www.eo-miners.eu). The goal of EO-MINERS is to "integrate new and existing Earth Observation tools to improve best practice in mining activities and to reduce the mining related environmental and societal footprint". Accordingly, the main objective of the current study was to develop hyperspectral-based means for the detection of small spectral changes and to relate these changes to possible degradation or reclamation indicators of the area under investigation. To ensure significant detection of small spectral changes, the temporal domain was investigated along with careful generation of reflectance information. Thus, intensive spectroradiometric ground measurements were carried out to ensure calibration and validation aspects during both overflights. The performance of these corrections was assessed using the Quality Indicators setup developed under a different FP7 project-EUFAR (http://www.eufar.net), which helped select the highest quality data for further work. This approach allows direct distinction of the real information from noise. The reflectance images were used as input for the application of spectral-based change-detection algorithms and indices to account for small and reliable changes. The related algorithms were then developed and applied on a pixel-by-pixel basis to map spectral changes over the space of a year. Using field spectroscopy and ground truth measurements on both overpass dates, it was possible to explain the results and allocate spatial kinetic processes of the environmental changes during the time elapsed between the flights. It was found, for instance, that significant spectral changes are capable of revealing mineral processes, vegetation status and soil formation long before these are apparent to the naked eye. Further study is being conducted under the above initiative to extend this approach to other mining areas worldwide and to improve the robustness of the developed algorithm.
\end{abstract}

Keywords: spectral change, coal mining, change detection, HyMap

\section{INTRODUCTION}

Among anthropogenic environmental hazards, mining activities have a most serious immediate impact on their local surroundings. Formation of sinkholes, loss of biodiversity, dust, erosion, slope instability, and contamination of soil, 
groundwater and surface water by chemicals from mining processes are some of the harmful effects encountered in these areas.

Mining areas are some of the most rapidly changing areas on earth. The obvious changes are caused by extraction of soil and its deposition in waste dumps. This causes changes in terrain altitude and topsoil components, and mineralogical alterations caused by exposing deep compressed minerals to air, water and wind. In addition, indirect changes result from, for example, employment opportunities related to the mining industry (creating residential and social changes). Detecting and analyzing these various changes is of major importance for decision-makers, environmental-watchers and the local residents ${ }^{1}$.

Reflectance spectroscopy and imaging spectroscopy (IS), also known as hyperspectral remote sensing HSR or hyperspectral imaging, have been shown to provide good classification capabilities for minerals, soils, vegetation and various pollutants in different applications. Techniques for change detection from HSR data have been shown to be effective at detecting changes in a scene ${ }^{2}$. Among the various change-detection methods, we chose to focus on linear chronochrome $^{2}$ (LCC), linear covariance equalization ${ }^{3}$ (LCE), principal component analysis ${ }^{4}$ (PCA) and spectral angle mapper $^{5}$ (SAM). The aim of this paper is to compare the results obtained by these algorithms and to validate them against ground truth information. The area selected for this study was the open-pit Sokolov mining area in the Czech Republic, using the airborne HyMap sensor. In the first section, we provide a brief summary of the change-detection algorithms. Then we describe the study area and the methods used for data acquisition and preprocessing (atmospheric corrections and geo-rectification). In the third section, the change-detection results and capabilities are presented.

\subsection{Change detection based on linear transformation and $\mathrm{RX}$ detector}

Schaum and Stocker ${ }^{6}$ introduced two different methods, based on the same process, to apply a linear estimate of one image taken at time 1 , denoted by $\mathrm{x}$, using another image of the same scene taken at a different time 2, denoted by $\mathrm{y}$. This linear transformation, regarded as step 1, is followed by an RX anomaly detector ${ }^{7}$, in step 2 .

They assume the use of two hyperspectral images of the same (or similar) area. The set of vector data $\{\mathrm{x}\}$ represents (mean-centered) sensor digital data collected at time 1. Each element of the set corresponds to a different pixel, and each component of $\mathrm{x}$ corresponds to a sensor estimate of spectral radiance. A similar set $\{\mathrm{y}\}$ defines the data from time 2 . The second-order statistics of these data sets are described by the covariance matrices:

$$
X \equiv\left\langle x x^{t}\right\rangle, \quad Y \equiv\left\langle y y^{t}\right\rangle, \quad C \equiv\left\langle y x^{t}\right\rangle
$$

in which the brackets indicate "expected value". These second-order statistics are assumed to have been derived from time 1 and 2 data collections.

With any linear estimate

$$
y \approx L x
$$

We can define the error by

$$
\varepsilon=(y-L x)
$$

and the error matrix by

$$
E=\left\langle(y-L x)(y-L x)^{t}\right\rangle
$$

For step 2, we note that, in general, $\mathrm{RX}$ applied to a data set $\{\mathrm{x}\}$ consists of comparing the scalar statistic to a fixed threshold 


$$
d_{R X}=x^{t} X^{-1} x
$$

For the change-detection application, $\mathrm{x}$ becomes the error signal, and $\mathrm{d}_{\mathrm{RX}}$ takes the form

$$
d=\varepsilon^{t} E^{-1} \varepsilon
$$

The process described above applies to both methods; the difference between them is the linear transformation $\mathrm{L}$. The first method is called chronochrome ${ }^{2}$ and the second one covariance equalization ${ }^{3}$. Both are described in the following subsections.

\subsection{Chronochrome change detection ${ }^{2}$ (LCC)}

The minimum mean square error (MMSE) solution described by Schaum and Stocker ${ }^{2}$ for the linear transformation is given by:

$$
L_{C C}=C X^{-1}
$$

known in hyperspectral remote sensing as the chronochrome (CC) transformation, and mathematically as a matrix Wiener filter.

Combining (3) and (7) we get:

$$
\varepsilon_{c c}=\left(y-C X^{-1} x\right)
$$

and from Equations (6) and (8), it follows that the chronochrome detector is:

$$
d_{c c}=\varepsilon_{c c}^{t}\left\langle\left(y-C X^{-1} x\right)\left(y-C X^{-1} x\right)^{t}\right\rangle^{-1} \varepsilon_{c c}
$$

We can simplify this further to:

$$
d_{c c}=\varepsilon_{c c}^{t}\left(Y-C X^{-1} C^{t}\right)^{-1} \varepsilon_{c c}
$$

Since the covariance matrix $\mathrm{C}$ requires co-located pairs of pixels from the $\mathrm{x}$ and $\mathrm{y}$ sets in the covariance computation, when the images are not perfectly aligned, this error will add to error caused by comparing misregistered pixels.

\subsection{Change detection based on covariance equalization ${ }^{3}$ (LCE)}

LCE is an alternative to the LCC transformation that does not require image registration in the process of calculating the linear transformation L. In this case, LCE will be necessarily suboptimal compared with LCC which is the least error estimator, but will not be sensitive to image registration.

The defining equation for the LCE transformation is:

$$
L_{C E} X L_{C E}^{t}=Y
$$

Schaum and Stocker ${ }^{3}$ presented the general solution as:

$$
L_{C E}=Y^{1 / 2} R X^{-\not / 2}
$$

where $\mathrm{R}$ is a general rotation matrix. The authors indicated that the simple choice of $\mathrm{R}=\mathrm{I}$ shows good results, which leads us to the following error signal by using (3) and (12): 


$$
\varepsilon_{c E}=\left(y-Y^{\frac{1}{2}} X^{-\frac{1}{2}} x\right)
$$

Finally, the covariance equalization change detector is given by:

$$
d_{c E}=\varepsilon_{c E}^{t} E_{c E}^{-1} \varepsilon_{c E}
$$

where

$$
E_{C E} \equiv\left\langle\varepsilon_{C E} \varepsilon_{C E}^{t}\right\rangle
$$

\subsection{Change detection using principal component analysis (PCA)}

PCA is probably the most common linear transformation technique. The main principle of the PCA approach ${ }^{4}$ is to use a set of images as input and reorganize them via a linear transformation, such that the output images are uncorrelated. The new coordinate system for the data is projected such that the greatest variance lies on the first axis or the first principal component and the second greatest variance on the second axis. This technique is usually used to reduce the number of spectral bands or in compression schemes. In change-detection studies, the consequence of this linearization is that unchanged pixels or common information shared by a pair of images are expected to lie in a narrow elongated cluster along a principal axis equivalent to the first component (PC1). In contrast, pixels containing a change will be more unique in their spectral appearance and are expected to lie far away from this axis (PC2).

Thus, the magnitude of a change is quantified by the magnitude of PC2 of the overall zero-mean covariance matrix. To obtain the principal components, the PCA process is conducted using Singular Value Decomposition (SVD) over the following matrix:

$$
\mathbf{C}_{i}=\frac{1}{n} \sum_{\mathbf{x}}\left(\mathbf{x}_{i}-\mathbf{m}_{i}\right)\left(\mathbf{x}_{i}-\mathbf{m}_{i}\right)^{\mathrm{T}}
$$

where

$$
\mathbf{x}_{i}=\left[x_{i}\left(T_{1}\right), x_{i}\left(T_{2}\right)\right]^{\mathrm{T}}
$$

$\mathrm{x}_{\mathrm{i}}\left(\mathrm{T}_{1}\right)$ and $\mathrm{x}_{\mathrm{i}}\left(\mathrm{T}_{2}\right)$ are vector representations of the twice-differing images and

$$
\mathbf{m}_{i}=\frac{1}{n} \sum \mathbf{x}_{i}
$$

\subsection{Spectral Angle Mapper ${ }^{5}$ (SAM)}

SAM is a well-known technique that is used in many HRS applications (supervised classification oriented). Since every pixel in the image has numerous wavelength values, it is often considered a vector pixel. The angle between two corresponding vector pixels in two images that relate to the same geographic location is calculated and inspected. Large angle values indicate a significant change and small values indicate minor changes.

$$
\operatorname{SAM}(x, y)=\cos ^{-1}\left(\frac{(\vec{x}, \vec{y})}{\|\vec{x}\|\|\vec{y}\|}\right)
$$

SAM is a very robust method for albedo variations, making it useful when mosaicking several flight-line images with different albedo values on the boundaries of the flight lines. It is also easy to implement and does not require highly intensive computation. 


\section{MATERIALS AND METHODS}

\subsection{The study site: Sokolov mining area}

The Sokolov basin (Figure 1), Oligocene to Miocene in age, is 8-9 km wide by $36 \mathrm{~km}$ long, with a total area of about $312 \mathrm{~km}^{2}$. It consists of $60 \%$ volcanic ejects resulting from faults and volcanic cones, and $40 \%$ sediments. It is bordered by a complex SW-NE faulting system and is cut by NW-SE faults. The central part of the basin is delimited to the north by the Lipnice faulting system and to the south by the Sokolov faulting system. Hydrothermal fluids have been circulating along the faults where silicifications and sulfides can be found, responsible for acid mine drainage (AMD). Lignite comprises three coal seams. The Josef coal seam represents the lowest seam, overlying the basement rocks. It is very rich in sulfur (up to 5\%) and arsenic (60-70 ppm). The Anežka seam is more recent than the Josef seam and is developed only in the western part of the basin. The Josef and Anežka seams have been exploited in particular in the Medard open pit. The Antonin seam is currently exploited in the Jiř́ open pit and it contains $1.1 \%$ sulfurs on average, whereas the marginal resources, containing locally up to $9 \%$ sulfurs together with arsenic, are not the object of mining. Thick (currently 90-130 m) overburden is represented by the clays of the Cypris Formation (Burdigalian), predominantly kaolinite at the base, then illite and montmorillonite (smectite) at the top. The Cypris Formation contains interbeds of limestone and volcaniclastics and is capped by a thin cover (1-5 m) of Quaternary loess loams.

At present, two coal mines remain active (Jiří, and Družba), whereas there are numerous abandoned (Lomnice, Sylvestr, Medard-Libík, etc.) open-pit mines and abandoned waste-rock dumps around the coal mining area (PVS Podkrušnohorská výsypka, Lítov, etc.) in Sokolov. Comprehensive field measurements $(2008,2009)$ confirmed that the study area is largely affected by AMD due to the significant presence of sulfur in the mining material (this is mainly from brown coal, which contains 5 to $8 \%$ pyrite, and hydrothermal deposits migrating along the fault system that borders the basin). AMD affects the mine waters in formerly exploited open pits. Besides the well-known acidification pattern resulting from AMD, a strong negative correlation between surface $\mathrm{pH}$ and the lignite-carbon-rich material has been observed. Furthermore, a very high correlation between this material and toxic trace elements such as mercury and arsenic was found when studying the material of the waste-rock dumps in Sokolov.

Over 100 sampling points distributed within the still active (Jiř́, Medard and Družba) and abandoned (Lítov, Lomnice, Sylvestr and PVS - Podkrušnohorská výsypka) open-pit mines were documented in the field. Particular attention was paid to the significantly AMD-affected areas in the abandoned mines. At the sampling points, reflectance measurements were collected under natural illumination conditions using a portable ASD FieldSpec $3 \circledR$ field spectroradiometer, with the spectrum of each ground point representing an average calculated from at least three point measurements distributed along the petrologically homogeneous material. Further spectra were obtained in the laboratory using artificial illumination conditions, a contact probe assembly and homogeneous samples taken from the surface (0-2 cm depth). Fifty selected points were obtained using both field and laboratory spectral measurements. All of the samples brought to the laboratory were dried and sieved to $<2 \mathrm{~mm}$. The abundance of trace elements was measured using a portable Innov- $\mathrm{x}$

Alpha RFA spectrometer. Thirty of the samples were further subjected to selected X-ray diffraction analysis (for mineral determination), and determinations of $\mathrm{pH}$, sulfur ( $\mathrm{S}$ total wt) and total organic carbon (TOC). The locations of the selected samples are marked in Figure 1. 


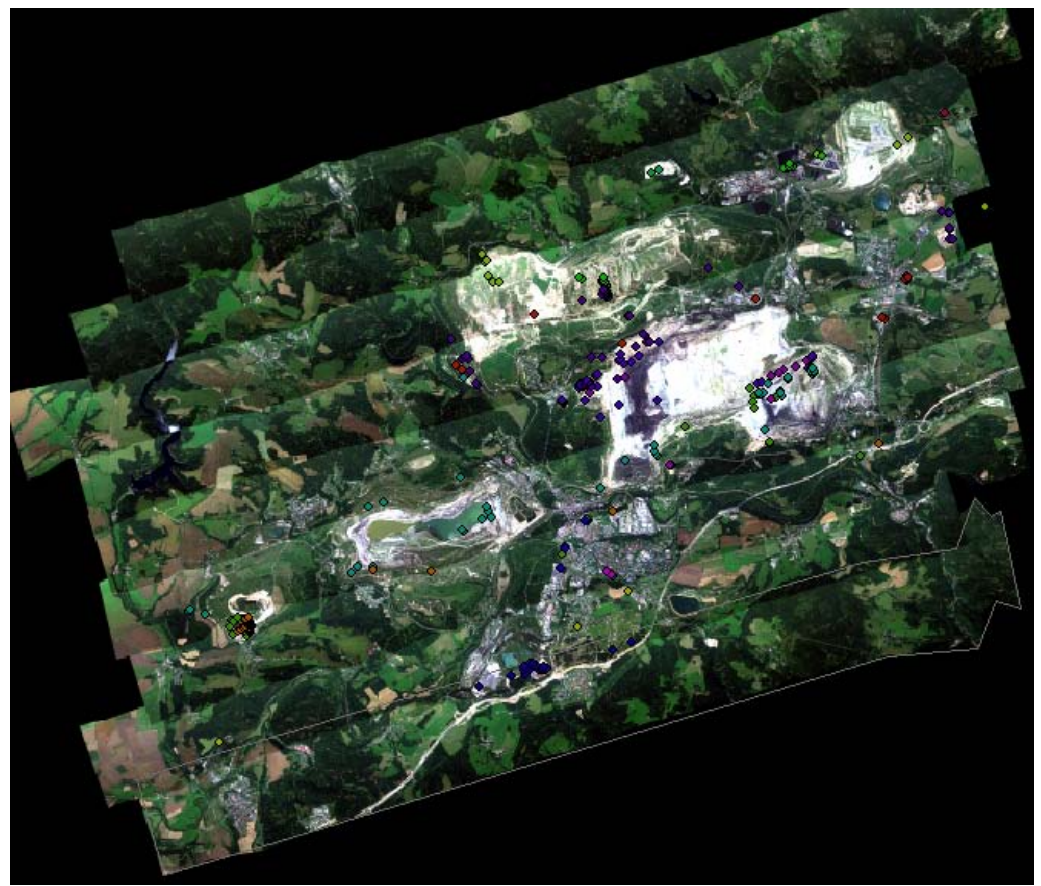

Figure 1. Sokolov area. Mosaic image (acquired in 2009) with sample locations.

\subsection{Data acquisition and processing}

Two HyMap images were acquired, in July 2009 and August 2010, over the Sokolov area. The 2009 flight campaign included 9 flight lines and the 2010 flight campaign 7 flight lines. The sensor configuration was set to 125 wavelengths across the 450-2500 nm spectral range. A ground team of 15 scientists and students collected the ground truth at the time of the overpasses. Atmospheric correction was performed using ATCOR ${ }^{8}$ code. Geo-rectification of the HyMap strips was done using PARGE software, whereas mosaicking of the rectified images was performed by ENVI software.

The change-detection algorithms described in section 1 were implemented using Matlab software in the MathWorks environment. Both ENVI and ArcGIS software were used to archive the data to a precise geographic projection. Ground geo-tagging of photos, ASD measurements and metadata description were performed with GeoSciTag software from Novospec Ltd., which exported the data to Google Earth and Excel spreadsheets. For simplicity (to conserve run time and computer memory), we applied the algorithms over a representative subset image. The selected area was part of the active mining processes indicated as a major source for possible environmental impact within the Sokolov area.

\section{RESULTS}

\subsection{Geo-rectification problems}

Although most of the image geo-rectification is aligned, there are places with geo-rectification errors. These errors can be a result of flight fluctuations, different target angle view and shading of targets, which cannot be corrected. These errors must be taken into account when performing change detection between two images to ensure that the changes detected by the algorithm are spectrally and not spatially oriented. This problem can be dealt with in different ways, such as downscaling or averaging the image. In this study, we chose to average the image with a sliding window of $\mathrm{P} \times \mathrm{P}$ dimensions where $\mathrm{P}$ can be determined by the geo-rectification error. Since most of the image has one pixel error in georectification, we used a 3 x 3 sliding window, which is one pixel error in each direction.

\subsection{Coal mining area: changes and interpretation}

As previously mentioned, an image subset was selected within the Sokolov active mining area shown in Figure 2. This figure provides the area along the basic strip (A), the georectified image (B) and the common area on a similar color composite. As noted from Figure 2C and D, significant changes occurred in the area during the studied year. 


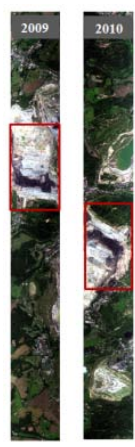

(A)

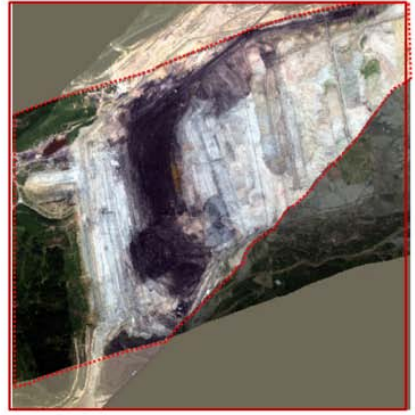

(B)

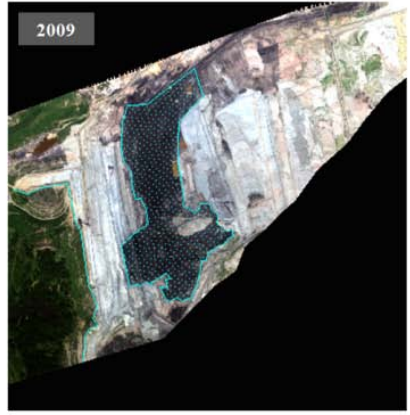

(C)

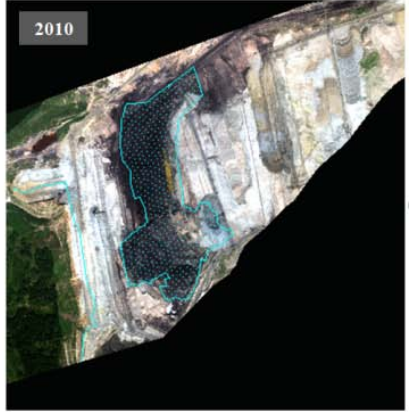

(D)

Figure 2. Subset of the Sokolov mining area as a test case. (A) 2009 and 2010 image strips. (B) Geo-rectification of the 2009 and 2010 strips; the overlapping area is demarcated by a red dotted line. (C) The coal-mining area in 2009 is demarcated with a cyan line. (D) The coal-mining area in 2010, where the mining progress is emphasized by comparing with the 2009 coal-mining area (cyan). The RGB images were composed from $634 \mathrm{~nm}, 543 \mathrm{~nm}$ and $466 \mathrm{~nm}$, respectively.

\subsection{Different detection capabilities}

Figure 3A shows the subscene further selected to apply the change-detection algorithms. Changes in area ii were detected by SAM, PCA and LCE, but not by LCC (B,C,E and D, respectively). It is interesting to note that the applied change-detection algorithms show different performance with respect to the degree of spectral changes occurring in different areas, even as pertains to major changes. This can be seen in Figure 4 for areas i and ii. In Figure 4B (area ii in Figure 3), the same extension trend, with movement of water ponds to the west, is visible with SAM, LCC and LCE (see Figure 3 B,D,E) but not with PCA (Figure 3C).

In these examples, the SAM algorithm shows the best intensity separation between the easily seen spatial changes. In addition, these examples show how spectral information contributes to the detection of changes using the selected algorithm over "easily seen" cases.
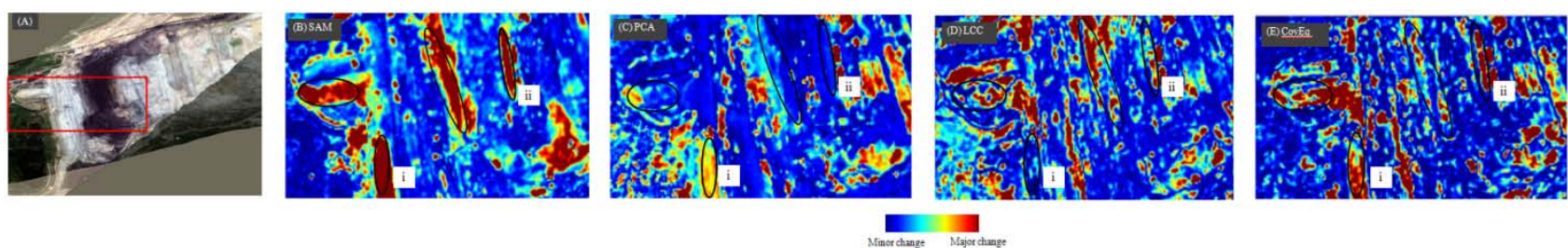

Figure 3. (A) The subset to which the change-detection algorithms were applied. (B-E) The algorithms SAM, PCA, LCC and LCE, respectively. The ellipses in (B-E) outline the areas changed due to the progress of mining, as mentioned in Figure 2.
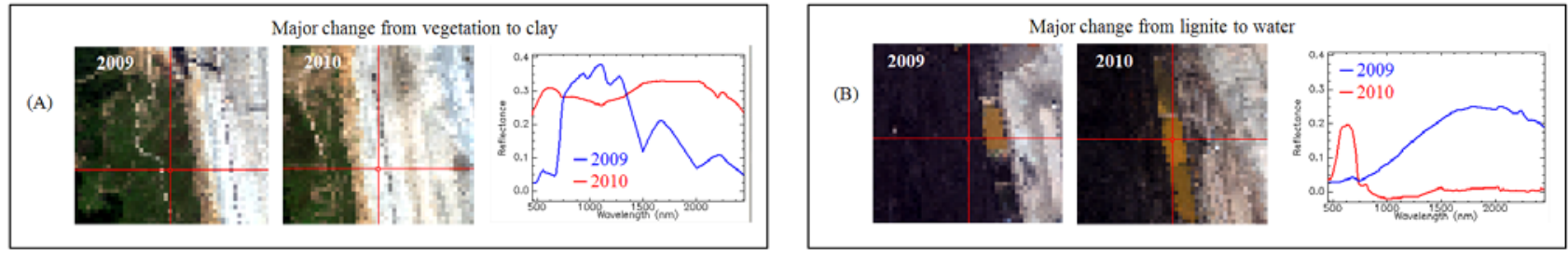

Figure 4. Examples of spectral changes over two areas. (A) and (B) correspond to ellipses $i$ and ii in Figure 3.

\subsection{Detection of changes which cannot be seen with the "naked eye"}

As the best-performing change-detection algorithm in the previous stages was SAM, we selected it to examine further "hot spots" not visible to the naked eye using a simple color composite as shown in Figure 1. Figure 5 shows the SAM's 
hotspots and spectral investigation of selected pixels within these spots. Significant spectral changes in selected pixels could be extracted by SAM, although no corresponding visible changes had been encountered. For example, changes from wet-clay or coal surface coverage in 2009 to water coverage in 2010 can be seen in Figure 5 A1 and A2. These materials are dark in the visible region but spectrally different when assessed by the entire sensor sensitivity. Moderate changes, such as in clay moisture (Figure 5 A3), and minor changes, such as in the amount of coal coverage (Figure 5 A4), appear as yellowish and blue areas. These examples show that a spectral-based algorithm can detect changes where a spatial-based algorithm cannot. Although these examples illustrate a very simple case, we believe that more spectralbased changes are occurring in the area and it is important that they be extracted.

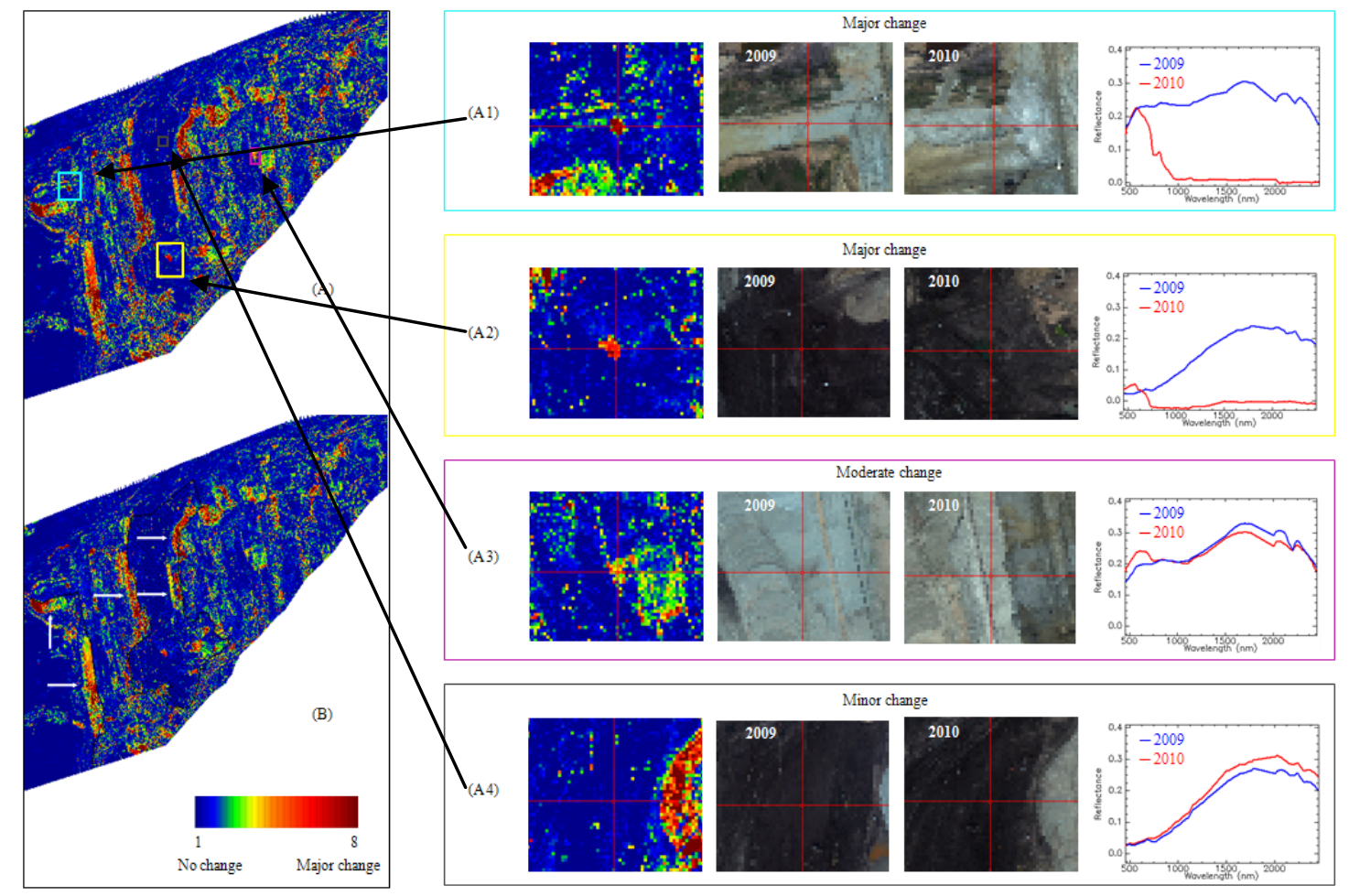

Figure 5. SAM output map emphasizing the pixels in which spectral changes have occurred. Examples of such changes are shown: (A1) from clay to water (cyan rectangular), (A2) from lignite to water (yellow rectangular), (A3) different clay condition (magenta rectangular), (A4) minor change (gray rectangular). White arrows in (B) point to changes due to the progress of mining mentioned in Figure 2.

\section{CONCLUSIONS AND FUTURE WORK}

Our aim in this study was to demonstrate the detection of spectral-based changes in coal mining areas that are not visible to the naked eye. The selected study area was the open-cast coal mine in Sokolov, Czech Republic, where two HSR campaigns were run in July 2009 and August 2010 using the HyMap sensor. In this study, we used several changedetection algorithms from the literature to check their performance, and implemented them on a subscene of the mining area. The algorithms were: linear chronochrome (LCC), linear covariance equalization (LCE), principal component analysis (PCA) and spectral angle mapper (SAM).

The selected algorithms detected most of the changes, some of them major. However, different detection capabilities were observed based on spectral information. The best-performing spectral-based algorithm was found to be SAM. This 
screening stage encouraged us to proceed with the SAM algorithm to reveal changes not visible to the naked eye. Some of the interesting changes that we found included changes from wet-clay and coal to water, which are considered very important for the mining companies as they suggest unstable slopes, and can alert the companies to the potential collapse of buildings, roads, natural surroundings and deep mining walls and to dangerous truck and human byways.

We intend to continue this research toward better implementation and probably modification of the SAM algorithm to detect small spectral-based changes. Although this study is focused on a mining area, we intend to extend our research area to waste dumps, re-cultivation areas, abandoned mines, natural vegetation and urban areas.

We investigated the faults of some of the algorithms that were implemented in this study to improve change-detection performance. In a preliminary test, we observed that the linear transformation of LCC and LCE might not work well on our data. The linear transformation attempts to bring one image closer to another and to overcome atmospheric correction and illumination differences, as well as other differences that are not due to inherent reflectance changes. However, it will provide good results when most of the data are unchanged. As the term 'most of the data' is not sufficiently well defined, we will try to develop a quantitative measure that will help determine whether a set of images is suitable for the linear transformation approach or not. This tool, we believe, will give scientists and engineers the ability to quickly assess the applicability of a change-detection approach without having to go through a trial-and-error stage. In addition, we believe that multi-stage change detection can produce better results in change-detection performance as well as in classification processes. The process will include several stages in which we will mask different categories of changes (both major and minor).

Acknowledgement

The present research is being undertaken within the framework of the FP7 Project (EO-MINERS, Grant Agreement $\mathrm{n}^{\circ}$ 244242), and the grant n²05/09/1989 funded by the Czech Science Foundation.

\section{REFERENCES}

[1] Thornton, I., "Impacts of mining on the environment; some local, regional and global issues," Applied Geochemistry 11(1-2), 355-361 (1996).

[2] Schaum, A., and Stocker, A., "Long-interval chronochrome target detection,” in Proceedings of the International Symposium on Spectral Sensing Research (1997).

[3] Schaum, A.P., and Stocker, A., "Hyperspectral change detection and supervised matched filtering based on covariance equalization,” in Proceedings of SPIE 5425, 77 (2004).

[4] Wiemker, R., Speck, A., Kulbach, D., Spitzer, H., and Bienlein, J., "Unsupervised robust change detection on multispectral imagery using spectral and spatial features," in Proceedings of the Third International Airborne Remote Sensing Conference and Exhibition 1, 640-647 (1997).

[5] Kruse, F.A., Lefkoff, A.B., Boardman, J.W., Heidebrecht, K.B., Shapiro, A.T., Barloon, P.J., and Goetz, A.F.H., "The spectral image processing system (SIPS) - interactive visualization and analysis of imaging spectrometer data," Remote Sensing of Environment 44(2-3), 145-163 (1993).

[6] Schaum, A., and Stocker, A., "Advanced algorithms for autonomous hyperspectral change detection,” in Applied Imagery Pattern Recognition Workshop, 2004. Proceedings. 33, 33-38 (2004).

[7] Reed, I.S., and Yu, X., "Adaptive multiple-band CFAR detection of an optical pattern with unknown spectral distribution,” IEEE Transactions on Acoustics, Speech and Signal Processing 38(10), 1760-1770 (1990).

[8] Richter, R., and Schlapfer, D., "Geo-atmospheric processing of airborne imaging spectrometry data. Part 2: atmospheric/topographic correction,” International Journal of Remote Sensing 23(13), 2631-2649 (2002). 